\title{
Soil chemical properties and mineral nutrition of Latvian accessions of Trifolium fragiferum, a crop wild relative plant species
}

\author{
Una Andersone-Ozola ${ }^{1}$, Astra Jēkabsone ${ }^{1}$, Andis Karlsons ${ }^{2}$, \\ Māris Romanovs' ${ }^{1}$, Gederts levinsh ${ }^{1 *}$
}

\author{
${ }^{1}$ Department of Plant Physiology, Faculty of Biology, University of Latvia, Jelgavas 1, Riga LV-1004, \\ Latvia \\ ${ }^{2}$ Institute of Biology, University of Latvia, Ojāra Vācieša 4, Riga LV-1004, Latvia \\ ${ }^{\star}$ Corresponding author, E-mail: gederts.ievins@lu.lv
}

\begin{abstract}
The aim of the present study was to compare soil chemical composition and aspects of mineral nutrition of several accessions from different geographically isolated sites in Latvia of Trifolium fragiferum L., a crop wild relative plant species. Eight geographically isolated micropopulations of $T$. fragiferum in Latvia were identified, and material was sampled for analysis of plant-available mineral nutrient concentrations in soil and mineral nutrient concentrations in leaf tissues. Micropopulations of T. fragiferum were associated with rivers, lakes and the sea, thus also outside typical coastal or inland-located salt-affected grassland habitats. Soil concentration of plant-available mineral nutrients showed significant variation between different sites, ranging from $59 \%$ for $\mathrm{N}$ to $193 \%$ for Ca. Variation in mineral element concentration in plant leaf tissues was relatively lower. Multivariate analysis showed that sites with similar localization on river shores or in coastal areas had similar characteristics, as these were grouped together according to both soil mineral nutrient availability as well as leaf tissue mineral element concentrations. T. fragiferum can be characterized as a species able to maintain mineral homeostasis in leaf tissues irrespective of large variability in soil plant-available mineral nutrient concentrations and different levels of soil $\mathrm{pH}$. Together with general resilience to environmental constraints, this allows to predict a relatively good conservation status of $T$. fragiferum in Latvia in spite of rather limited number and size of the geographically isolated micropopulations.
\end{abstract}

Key words: crop wild relatives, mineral nutrition, mutivariate analysis, soil mineral elements, strawberry clover, Trifolium fragiferum, wild plants.

Abbreviations: CWR, crop wild relatives; EC, electrical conductivity.

\section{Introduction}

A crop wild relative (CWR) plant species is defined as a "wild plant taxon that has an indirect use derived from its relatively close genetic relationship to a crop" (Maxted et al. 2006; Ford-Lloyd et al. 2011). CWRs constitute valuable genetic resource representing a potential source of variation in respect to environmental heterogeneity (Warschefsky et al. 2014) that can be used for breeding of new crop varieties better adapted to global climate change (Zhang et al. 2016; Prohens et al. 2017). Local inventory of CWR resources is an important step in evaluation of wild plant diversity and necessary conservation measures (Maxted et al. 2007).

Wild legume species are especially important as CWRs in the context of the necessity for increasing sustainability of agricultural production (Zhang et al. 2019). First, legumes are important as protein crops of high quality both for food and feed. Second, cultivation of legume species leads to increased soil nitrogen concentration through symbiosis with nitrogen-fixing rhizobacteria. Among legumes, clover
(Trifolium) species are the most important as forage crops (Ravagnani et al. 2012). There are several Trifolium species listed among the legume forages included in Annex I of the International Treaty on Plant Genetic Resources for Food and Agriculture aimed at conservation, exploration, collection, characterization, evaluation and documentation of plant genetic resources (FAO 2009). Economically, most important clover species today are Trifolium repens and Trifolium pratense, but several other species of the genus are important for cultivation in suboptimal conditions, or for use in research aiming to improve the major clover species (Ravagnani et al. 2012; Egan et al. 2021). However, the FAO list does not include strawberry clover, Trifolium fragiferum L., which is a species native for Europe, West Asia, Mediterranean region, and Middle East (Zohary, Heller 1984). T. fragiferum has been commercialized in Australia and is in use also in the USA and New Zealand (Nichols et al. 2012). Relatively high abiotic stress tolerance of the species, including ability to successfully produce on saline lands, justifies its attractiveness as a valuable forage 
crop for complicated agroecological conditions (Can et al. 2013; Andersone-Ozola et al. 2021).

T. fragiferum is a perennial semi-rosete plant with pronounced clonality, as creeping stolons are able to form adventitious roots at nodes in conditions of high humidity (Huber, Wiggerman 1997). In the Baltic Sea region, T. fragiferum is a characteristic species of the primary vegetation of the middle and upper geolittoral in the Festucetosum rubrae subassociation of the Juncetum gerardi association (Tyler 1969). According to ecological indicator values for Sweden, T. fragiferum is characterized as favoured by moderate salinity but not restricted to saline habitats (indicator value 3 of 5; Tyler et al. 2021). The species is regarded as both a characteristic as well as umbrella species of the European protected habitat "Boreal Baltic coastal meadows" (1630*; Rūsina 2013). In Latvia, T. fragiferum has a status of a legally protected species (Cabinet of Ministers of Latvia 2000).

In general, heterogeneity of plant-available mineral nutrient concentration in coastal habitats is one of the main extremely pronounced environmental characteristics in addition to seawater influence and sand accretion (Ievinsh 2006). Therefore, it is evident that coastal plant species need to possess physiological mechanisms of maintenance of mineral homeostasis in plant tissues on the background of spatially and temporally variable soil mineral nutrient concentrations. Previously, mineral nutrient dynamics and mycorrhizal status of $T$. fragiferum and several other rare halophytic species have been assessed in field conditions (Karlsons et al. 2017), and recently, comparison of abiotic stress tolerance of different coastal accessions of the species has been performed in controlled conditions (AndersoneOzola et al. 2021). However, there is no comparative information available on requirements of $T$. fragiferum to edaphic conditions and soil salinity in native habitats.
Therefore, having in mind a necessity for conservation of genetic resources of CWRs in protected areas (Maxted 2003), the aim of the present study was to compare soil chemical composition and aspects of mineral nutrition of several accessions from different geographically isolated sites of $T$. fragiferum in Latvia.

\section{Materials and methods}

\section{Sites with T. fragiferum}

During preliminary studies, seven geographically isolated micropopulations (sites) of Trifolium fragiferum L. in Latvia were identified (TF1 to TF7, Fig. 1 and Table 1). Within the present study, one additional site was established (TF2b). All sites where $T$. fragiferum plants are found represent relatively small areas of land, from only several hundreds of $\mathrm{m}^{2}$ as in the case of TF2, TF2b, TF4 to several thousand $\mathrm{m}^{2}$ as in the case of TF1. Distribution of individuals in sites is markedly uneven, and the number of plants ranges from about 10 individuals (sites TF2, TF3, TF7) up to several hundred (site TF1). However, more precise estimates have not been performed and are not reasonable given the clonal growth nature of the plants. All sites except TF4 are located within a shore zone of different natural waterbodies and rivers: Lake Liepāja (TF1), River Lielupe (TF2, TF2b), River Bullupe (branch of the River Lielupe at estuary; TF3), and the Gulf of Riga of the Baltic Sea (TF5, TF6, TF7). Several sites are obviously affected by salt water from the sea (TF1, TF2, TF5, TF6). Site TF4 is located in a previously urban industrial area on degraded land in the territory of the city of Rìga.

\section{Field sampling}

Field studies were performed within a week in the second half of August 2021. Three to four plots (about $1 \mathrm{~m}^{2}$ ) with

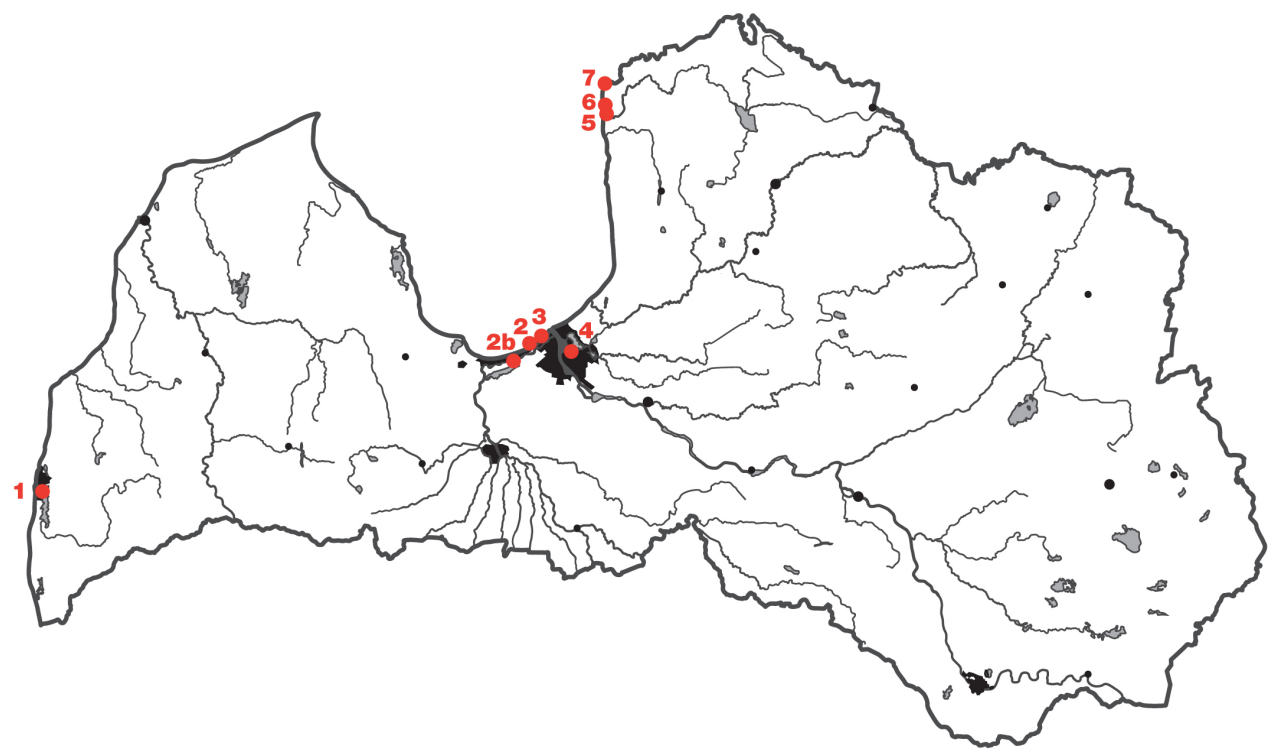

Fig. 1. Map of Latvia indicating geographically isolated sites of Trifolium fragiferum analyzed in the present study. 
Table 1. Geographically isolated micropopulations of Trifolium fragiferum in Latvia analyzed in the present study

\begin{tabular}{|c|c|c|c|c|}
\hline Site & Associated water reservoir & Habitat & Location & Coordinates \\
\hline $\mathrm{TF} 1$ & Lake Liepājas & $\begin{array}{l}\text { Salt-affected wet shore } \\
\text { meadow }\end{array}$ & City of Liepāja & $56^{\circ} 29^{\prime} 29^{\prime \prime} \mathrm{N}, 21^{\circ} 1^{\prime} 38^{\prime \prime} \mathrm{E}$ \\
\hline TF2 & River Lielupe & Salt-affected shore meadow & $\begin{array}{l}\text { City of Jūrmala, Lielupe, River } \\
\text { Lielupe Estuary }\end{array}$ & $57^{\circ} 0^{\prime} 11^{\prime \prime} \mathrm{N}, 23^{\circ} 55^{\prime} 56^{\prime \prime} \mathrm{E}$ \\
\hline $\mathrm{TF} 2 \mathrm{~b}$ & River Lielupe & Shore meadow & City of Jūrmala, Majori & $56^{\circ} 57^{\prime} 29^{\prime \prime} \mathrm{N}, 23^{\circ} 49^{\prime} 11^{\prime \prime} \mathrm{E}$ \\
\hline TF3 & River Bullupe & Shore meadow & $\begin{array}{l}\text { City of Rīga, Kurzeme District, } \\
\text { Island of Bullıu Sala, Vakarbullıi }\end{array}$ & $56^{\circ} 59^{\prime} 53^{\prime \prime} \mathrm{N}, 23^{\circ} 57^{\prime} 21^{\prime \prime} \mathrm{E}$ \\
\hline TF4 & - & Degraded urban land & City of Rīga, Vidzeme Suburb & $56^{\circ} 57^{\prime} 46^{\prime \prime} \mathrm{N}, 24^{\circ} 7^{\prime} 2^{\prime \prime} \mathrm{E}$ \\
\hline TF5 & The Gulf of Riga of the Baltic Sea & $\begin{array}{l}\text { Salt-affected wet coastal } \\
\text { meadow }\end{array}$ & $\begin{array}{l}\text { Salacgrīva Parish, Randu } \\
\text { Meadows }\end{array}$ & $57^{\circ} 49^{\prime} 51^{\prime \prime} \mathrm{N}, 24^{\circ} 20^{\prime} 12^{\prime \prime} \mathrm{E}$ \\
\hline TF6 & The Gulf of Riga of the Baltic Sea & $\begin{array}{l}\text { Salt-affected wet coastal } \\
\text { meadow }\end{array}$ & $\begin{array}{l}\text { Salacgrīva Parish, Randu } \\
\text { Meadows }\end{array}$ & $57^{\circ} 50^{\prime} 9^{\prime \prime} \mathrm{N}, 24^{\circ} 20^{\prime} 15^{\prime \prime} \mathrm{E}$ \\
\hline TF7 & The Gulf of Riga of the Baltic Sea & Dry coastal medow & Town of Ainaži & $57^{\circ} 52^{\prime} 8^{\prime} \mathrm{N}, 24^{\circ} 21^{\prime} 10^{\prime \prime} \mathrm{E}$ \\
\hline
\end{tabular}

T. fragiferum individuals were established within each site, located at least $10 \mathrm{~m}$ apart. At each plot of every site, one soil sample in the root zone (0 to $20 \mathrm{~cm}$ depth) was taken for laboratory analysis. Each soil sample (2 L) consisted of thoroughly mixed five subsamples. Leaves of $T$. fragiferum (about $5 \mathrm{~g}$ ) were collected for mineral element analysis at three plots for each site and placed in plastic ziplock bags. In the laboratory, leaves were separated in blades and petioles, and dried in an oven for $72 \mathrm{~h}$. Dried plant material were used for chemical analysis.

\section{Chemical analysis}

Plant tissues were ground into a fine powder using a ball mill. Plant tissue test solution was prepared by dry ashing with $\mathrm{HNO}_{3}$ vapor and re-dissolving in a $3 \% \mathrm{HCl}$ solution (Rinkis et al. 1987). The test solution was used for the determination of $\mathrm{N}, \mathrm{P}, \mathrm{K}, \mathrm{Ca}, \mathrm{Mg}, \mathrm{S}, \mathrm{Fe}, \mathrm{Mn}, \mathrm{Zn}, \mathrm{Cu}, \mathrm{Mo}$ and $\mathrm{B}$ in all plant tissue samples. The levels of $\mathrm{K}, \mathrm{Ca}, \mathrm{Mg}$, $\mathrm{Fe}, \mathrm{Cu}, \mathrm{Zn}, \mathrm{Mn}, \mathrm{Na}$ and $\mathrm{Pb}$ were estimated by a microwave plasma atomic emission spectrometer 4210 Agilent Technologies (Sreenivasulu et al. 2017). Levels of N, P, B was determine by colorimetry, $\mathrm{S}$ by visually nephelometric or turbidimetric method, and $\mathrm{K}$ by a flame photometer (Jenwey PFP7, air propane-butane flame). $\mathrm{Cl}$ concentration was determined by $\mathrm{AgNO}_{3}$ titration. All spectroscopic, colorimetric or photometric and titration determinations were performed in triplicate.

Soil samples were cooled at $4{ }^{\circ} \mathrm{C}$ to stop further nitrification and dried at $35^{\circ} \mathrm{C}$ to air-dry condition, then sieved through a $2 \mathrm{~mm}$ sieve. To determine plant-available mineral element ( $, \mathrm{P}, \mathrm{K}, \mathrm{Ca}, \mathrm{Mg}, \mathrm{S}, \mathrm{Fe}, \mathrm{Mn}, \mathrm{Zn}, \mathrm{Cu}, \mathrm{B}$, $\mathrm{Na}, \mathrm{Pb}$ ) concentration, soil samples were extracted using $1 \mathrm{M} \mathrm{HCl}$ (soil-extractant mixture 1:5 v/v). Oxidation of soil extract with concentrated $\mathrm{HNO}_{3}, \mathrm{H}_{2} \mathrm{O}_{2}$ and $\mathrm{HClO}_{4}$ was performed for the determination of $\mathrm{P}, \mathrm{S}$, and Mo. All nutrients were analyzed using the same procedures as in the case of plant samples.

In addition to the contents of mineral elements, two characteristics of the soil were identified, i.e. the soil active reaction $(\mathrm{pH})$ and total soluble salts concentration as electrical conductivity (EC). Soil pH was measured using a $\mathrm{pH}$ meter Basic Meter PB-20 in $1 \mathrm{M} \mathrm{KCl}$ extract. Soil electrical conductivity, which is a measure of the total soluble salt concentration, was determined by conductivity meter Hanna EC 215 in soil extract with distilled water.

\section{Data analysis}

Results were analyzed by KaleidaGraph (v. 5.0, Synergy Software, USA). Statistical significance of differences between sites was evaluated by one-way ANOVA using post-hoc analysis with minimum significant difference. Principal component analysis, heat map generation and cluster analysis were performed by a freely available web programme ClustVis (http://biit.cs.ut.ee/clustvis/; Metsalu, Vilo 2015). Hierarchial clusters were generated by average linkage method with correlation distance.

\section{Results}

The lowest soil $\mathrm{pH}$ level measured in $\mathrm{KCl}$ extract was in $T$. fargiferum site TF1, followed by TF3 (Table 2). Higher $\mathrm{pH}$ level occurred in TF6, TF7, TF5, and TF2b, reaching the highest values in TF4 and TF2. The highest level of EC in soil was in TF1, which was about four times that in TF5 and TF6. Statistically similar mean EC values were observed for the other sites.

Soil concentration of plant-available mineral nutrients showed significant differences between sites (Table 2), in general reflecting high variation of edaphic characteristics (Table 3). Two macroelements, $\mathrm{N}$ and $\mathrm{P}$, were relatively less variable in comparison to the other elements (Table 3). Three sites (TF2, TF2b, TF3) were extremely poor in $\mathrm{N}$, followed by three sites with medium concentration values (TF4, TF5, TF7), and the highest values were in two sites (TF1 and TF6; Table 2). Site TF4 was extremely poor in $\mathrm{P}$, four sites had medium concentration values (TF1, 
Table 2. Soil characteristics and plant-available mineral element concentrations $\left(\mathrm{mg} \mathrm{L}^{-1}\right)$ at Trifolium fragiferum sites. Data are means \pm SE from 3 to 4 samples at each site. Different letters within rows indicate statistically significant differences $(p<0.05)$. EC, electrical conductivity

\begin{tabular}{|c|c|c|c|c|c|c|c|c|}
\hline $\begin{array}{l}\text { Element } \\
\text { (unit) }\end{array}$ & TF1 & TF2 & TF2b & TF3 & TF4 & TF5 & TF6 & TF7 \\
\hline $\begin{array}{l}\mathrm{pH}_{\mathrm{KCl}} \\
\text { (units) }\end{array}$ & $6.53 \pm 0.43 \mathrm{~d}$ & $8.7 \pm 0.2 \mathrm{a}$ & $\begin{array}{c}7.78 \pm 0.13 \\
a b c\end{array}$ & $6.78 \pm 0.17 \mathrm{~cd}$ & $8.65 \pm 0.09 a b$ & $\begin{array}{c}7.70 \pm 0.04 \\
a b c\end{array}$ & $\begin{array}{c}7.07 \pm 0.17 \\
\mathrm{~cd}\end{array}$ & $\begin{array}{c}7.35 \pm 0.02 \\
\text { bcd }\end{array}$ \\
\hline $\begin{array}{l}\mathrm{EC}(\mathrm{mS} \\
\left.\mathrm{cm}^{-1}\right)\end{array}$ & $8.94 \pm 1.18 \mathrm{a}$ & $0.36 \pm 0.06 \mathrm{c}$ & $0.36 \pm 0.09 c$ & $0.60 \pm 0.37 c$ & $0.59 \pm 0.05 c$ & $2.18 \pm 0.50 \mathrm{~b}$ & $2.51 \pm 0.71 b$ & $0.21 \pm 0.03 c$ \\
\hline $\mathrm{N}$ & $72 \pm 4 \mathrm{a}$ & $16 \pm 3 c$ & $17 \pm 1 c$ & $21 \pm 1 c$ & $41 \pm 9 b$ & $47 \pm 3 b$ & $82 \pm 14 a$ & $39 \pm 4 b$ \\
\hline $\mathrm{P}$ & $129 \pm 38 \mathrm{~d}$ & $139 \pm 21 \mathrm{~d}$ & $165 \pm 3 d$ & $149 \pm 20 \mathrm{~d}$ & $31 \pm 13 \mathrm{e}$ & $352 \pm 19 b$ & $228 \pm 19 c$ & $458 \pm 11 \mathrm{a}$ \\
\hline K & $130 \pm 21 b$ & $24 \pm 6 \mathrm{~d}$ & $24 \pm 2 \mathrm{~d}$ & $33 \pm 8 \mathrm{~cd}$ & $566 \pm 103 \mathrm{a}$ & $55 \pm 15 c$ & $93 \pm 23 b$ & $12 \pm 2 \mathrm{e}$ \\
\hline $\mathrm{Ca}$ & $4800 \pm 890 \mathrm{~b}$ & $2327 \pm 873$ & $2580 \pm 81 \mathrm{~d}$ & $558 \pm 89 \mathrm{e}$ & $46735 \pm 644 \mathrm{a}$ & $2447 \pm 222 \mathrm{~cd}$ & $2658 \pm 95 \mathrm{~d}$ & $2708 \pm 13 c$ \\
\hline $\mathrm{Mg}$ & $1186 \pm 154 b c$ & $506 \pm 84$ & $1270 \pm 46 b$ & $143 \pm 38 d$ & $13698 \pm 788 a$ & $1016 \pm 137$ & $909 \pm 114 c$ & $1126 \pm 5 c$ \\
\hline S & $588 \pm 16 \mathrm{a}$ & $8 \pm 1 \mathrm{~d}$ & $15 \pm 3 c$ & $13 \pm 5 \mathrm{~cd}$ & $105 \pm 20 \mathrm{~b}$ & $70 \pm 21 \mathrm{~b}$ & $101 \pm 38 b$ & $12 \pm 1 c$ \\
\hline $\mathrm{Fe}$ & $1124 \pm 194 b$ & $299 \pm 73 c$ & $152 \pm 7 d$ & $201 \pm 59 d$ & $489 \pm 56 c$ & $626 \pm 233 c$ & $1758 \pm 245 a$ & $350 \pm 13 c$ \\
\hline $\mathrm{Mn}$ & $31 \pm 9 b$ & $15 \pm 6 c$ & $14 \pm 1 \mathrm{c}$ & $12 \pm 6 c$ & $107 \pm 2 \mathrm{a}$ & $26 \pm 7 \mathrm{~b}$ & $106 \pm 23 \mathrm{a}$ & $27 \pm 1 b$ \\
\hline $\mathrm{Zn}$ & $36.9 \pm 7.6 \mathrm{a}$ & $3.7 \pm 0.2 c$ & $2.9 \pm 0.9 c$ & $4.9 \pm 2.6 c$ & $8.1 \pm 1.3 b$ & $1.3 \pm 0.1 \mathrm{~d}$ & $11.2 \pm 2.3 \mathrm{~b}$ & $1.6 \pm 0.1 \mathrm{~d}$ \\
\hline $\mathrm{Cu}$ & $4.4 \pm 1.4 \mathrm{a}$ & $0.5 \pm 0.2 \mathrm{~cd}$ & $0.8 \pm 0.1 c$ & $0.6 \pm 0.2 c$ & $2.9 \pm 0.5 \mathrm{a}$ & $0.6 \pm 0.1 c$ & $2.0 \pm 0.4 \mathrm{ab}$ & $0.3 \pm 0.0 \mathrm{~d}$ \\
\hline B & $3.60 \pm 0.39 a$ & $0.17 \pm 0.03 \mathrm{~d}$ & $0.27 \pm 0.07 \mathrm{~d}$ & $0.83 \pm 0.09 c$ & $0.10 \pm 0.00 \mathrm{~d}$ & $1.23 \pm 0.03 b$ & $1.73 \pm 0.49 b$ & $0.15 \pm 0.05 \mathrm{~d}$ \\
\hline $\mathrm{Na}$ & $1114 \pm 180 \mathrm{a}$ & $57 \pm 13 c$ & $44 \pm 14 \mathrm{~d}$ & $64 \pm 4 c$ & $65 \pm 2 c$ & $357 \pm 87 \mathrm{~b}$ & $484 \pm 48 b$ & $22 \pm 2 \mathrm{e}$ \\
\hline $\mathrm{Cl}$ & $2008 \pm 436 \mathrm{a}$ & $23 \pm 9 d$ & $43 \pm 18 d$ & $99 \pm 9 c$ & $9 \pm 1 \mathrm{e}$ & $527 \pm 137 b$ & $508 \pm 147 b$ & $8 \pm 0$ e \\
\hline $\mathrm{Pb}$ & $17.2 \pm 4.5 \mathrm{a}$ & $1.8 \pm 0.3 \mathrm{~d}$ & $2.0 \pm 0.1 \mathrm{~d}$ & $2.3 \pm 0.6 \mathrm{~d}$ & $6.5 \pm 0.8 \mathrm{~b}$ & $3.2 \pm 1.2 \mathrm{~cd}$ & $5.0 \pm 1.6 \mathrm{bc}$ & $2.1 \pm 0.4 \mathrm{~d}$ \\
\hline
\end{tabular}

TF2, TF2b, TF3), and the highest vales were in TF6, TF5 and TF7. The concentration range for $\mathrm{K}$ also was highly pronounced (Table 3), with extremely low concentration in TF7 and low in TF2, TF2b, TF3 (Table 2). Higher K concentration occurred in the order TF5 $<$ TF $6<$ TF 1 , with extremely high concentration in TF4. Two other macroelements, $\mathrm{Ca}$ and $\mathrm{Mg}$, also had extremely high concentrations in TF4, with lowest levels in TF3 (Table 2 ), and their range showed extreme variability between the sites (Table 3). Another macroelement, S, showed

Table 3. Comparison of soil and plant mineral element concentrations found in the present study for Trifolium fragiferum with literature data on substrate optimum concentrations for legumes (Osvalde 2017) and optimum leaf concentrations for Trifolium repens (Schwab et al. 2007). CV, coefficient of variation

\begin{tabular}{|c|c|c|c|c|c|c|c|c|}
\hline \multirow[t]{2}{*}{ Element } & \multicolumn{2}{|c|}{ Soils } & \multirow{2}{*}{$\begin{array}{c}\text { Optimum } \\
\text { in inert } \\
\text { substrate for } \\
\text { legumes (mg } \\
\left.\mathrm{L}^{-1}\right)\end{array}$} & \multicolumn{2}{|c|}{ Leaf petioles } & \multicolumn{2}{|c|}{ Leaf blades } & \multirow{2}{*}{$\begin{array}{l}\text { Optimum } \\
\text { for } \\
\text { cultivated } \\
\text { white clover } \\
\text { in leaves }\end{array}$} \\
\hline & $\begin{array}{c}\text { Range } \\
\left(\mathrm{mg} \mathrm{L}^{-1}\right)\end{array}$ & CV (\%) & & Range & CV (\%) & Range & CV (\%) & \\
\hline & & & & $\left(\mathrm{g} \mathrm{kg}^{-1}\right)$ & & $\left(\mathrm{g} \mathrm{kg}^{-1}\right)$ & & $\left(\mathrm{g} \mathrm{kg}^{-1}\right)$ \\
\hline $\mathrm{N}$ & $16-82$ & 59 & $60-80$ & $16-25$ & 16 & $30-45$ & 13 & $45-50$ \\
\hline $\mathrm{P}$ & $31-458$ & 66 & $40-60$ & $1.1-2.9$ & 32 & $1.7-3.2$ & 18 & $3.6-4.5$ \\
\hline $\mathrm{K}$ & $24-130$ & 159 & 150 & $15-31$ & 28 & $6-15$ & 24 & $20-25$ \\
\hline $\mathrm{Ca}$ & $558-46735$ & 193 & $200-250$ & $2-15$ & 39 & $9-16$ & 22 & $5-10$ \\
\hline $\mathrm{Mg}$ & $143-13698$ & 183 & 60 & $3.3-5.6$ & 20 & $3.3-5.0$ & 15 & $2-3$ \\
\hline S & $8-588$ & 172 & 70 & n.d. & n.d. & n.d. & n.d. & $2.5-5.0$ \\
\hline $\mathrm{Cl}$ & $8-2008$ & 170 & - & $13-53$ & 38 & $2.0-8.9$ & 46 & - \\
\hline \multirow[t]{2}{*}{$\mathrm{Na}$} & $22-1112$ & 138 & - & $1.3-23.5$ & 50 & $0.8-7.6$ & 42 & - \\
\hline & & & & $\left(\mathrm{mg} \mathrm{kg}^{-1}\right)$ & & $\left(\mathrm{mg} \mathrm{kg}^{-1}\right)$ & & $\left(\mathrm{mg} \mathrm{kg}^{-1}\right)$ \\
\hline $\mathrm{Fe}$ & $49-1758$ & 88 & $5-10$ & $32-115$ & 48 & $69-104$ & 13 & $25-100$ \\
\hline $\mathrm{Cu}$ & $0.5-4.4$ & 97 & $0.3-0.4$ & $6-9$ & 14 & $9-12$ & 13 & $25-100$ \\
\hline $\mathrm{Zn}$ & $1.3-36.9$ & 134 & 1.0 & $27-56$ & 30 & $60-95$ & 20 & $15-25$ \\
\hline $\mathrm{Mn}$ & $12-107$ & 96 & $2.0-3.0$ & $19-67$ & 57 & $32-67$ & 16 & $5-8$ \\
\hline B & $0.1-3.6$ & 119 & $0.2-0.3$ & n.d. & n.d. & n.d. & n.d. & $25-50$ \\
\hline $\mathrm{Pb}$ & $2-17$ & 104 & - & $8.6-10.8$ & 8 & $7.7-8.9$ & 5 & - \\
\hline
\end{tabular}


Table 4. Mineral element concentrations in leaf blades of Trifolium fragiferum from different sites. Data are means \pm SE from 3 samples from each site. Different letters within rows indicate statistically significant differences $(p<0.05)$

$\begin{array}{lcccccccc}\begin{array}{l}\text { Element } \\ \text { (unit) }\end{array} & \text { TF1 } & \text { TF2 } & \text { TF2b } & \text { TF3 } & \text { TF4 } & \text { TF5 } & \text { TF6 } & \text { TF7 } \\ \mathrm{N}\left(\mathrm{g} \mathrm{kg}^{-1}\right) & 42.8 \pm 0.3 \mathrm{a} & 45.0 \pm 0.4 \mathrm{a} & 43.6 \pm 0.4 \mathrm{a} & 36.0 \pm 2.0 \mathrm{~b} & 30.2 \pm 1.8 \mathrm{c} & 37.2 \pm 2.0 \mathrm{~b} & 41.6 \pm 0.6 \mathrm{ab} & 45.0 \pm 0.8 \mathrm{a} \\ \mathrm{P}\left(\mathrm{g} \mathrm{kg}^{-1}\right) & 3.2 \pm 0.2 \mathrm{a} & 2.5 \pm 0.7 \mathrm{bc} & 3.0 \pm 1.0 \mathrm{ab} & 2.6 \pm 0.2 \mathrm{~b} & 2.9 \pm 0.3 \mathrm{~b} & 1.7 \pm 0.2 \mathrm{c} & 2.3 \pm 0.3 \mathrm{bc} & 2.7 \pm 0.5 \mathrm{~b} \\ \mathrm{~K}\left(\mathrm{~g} \mathrm{~kg}^{-1}\right) & 15.0 \pm 0.9 \mathrm{a} & 11.2 \pm 0.7 \mathrm{bc} & 10.6 \pm 0.4 \mathrm{c} & 9.4 \pm 2.8 \mathrm{bcd} & 12.5 \pm 0.7 \mathrm{~b} & 6.3 \pm 0.5 \mathrm{~d} & 10.1 \pm 1.0 \mathrm{c} & 9.7 \pm 0.8 \mathrm{c} \\ \mathrm{Ca}\left(\mathrm{g} \mathrm{kg}^{-1}\right) & 10.2 \pm 1.0 \mathrm{~b} & 10.0 \pm 0.8 \mathrm{~b} & 11.7 \pm 0.2 \mathrm{~b} & 8.6 \pm 0.2 \mathrm{c} & 8.6 \pm 0.6 \mathrm{c} & 15.7 \pm 0.9 \mathrm{a} & 9.5 \pm 1.0 \mathrm{bc} & 11.2 \pm 1.0 \mathrm{~b} \\ \mathrm{Mg}\left(\mathrm{g} \mathrm{kg}^{-1}\right) & 3.3 \pm 0.3 \mathrm{~b} & 5.0 \pm 0.4 \mathrm{a} & 4.8 \pm 0.1 \mathrm{a} & 4.9 \pm 0.6 \mathrm{a} & 4.5 \pm 0.1 \mathrm{a} & 4.9 \pm 0.3 \mathrm{a} & 3.4 \pm 0.1 \mathrm{~b} & 4.2 \pm 0.4 \mathrm{a} \\ \mathrm{Fe}\left(\mathrm{mg} \mathrm{kg}^{-1}\right) & 104 \pm 7 \mathrm{a} & 79 \pm 4 \mathrm{bc} & 84 \pm 7 \mathrm{ab} & 83 \pm 6 \mathrm{~b} & 84 \pm 1 \mathrm{~b} & 69 \pm 7 \mathrm{c} & 89 \pm 8 \mathrm{a} & 70 \pm 4 \mathrm{c} \\ \mathrm{Mn}\left(\mathrm{mg} \mathrm{kg}^{-1}\right) & 51 \pm 7 \mathrm{~b} & 33 \pm 3 \mathrm{~d} & 40 \pm 1 \mathrm{c} & 32 \pm 4 \mathrm{~d} & 41 \pm 1 \mathrm{c} & 48 \pm 7 \mathrm{bc} & 67 \pm 2 \mathrm{a} & 43 \pm 2 \mathrm{bc} \\ \mathrm{Zn}\left(\mathrm{mg} \mathrm{kg}^{-1}\right) & 86 \pm 11 \mathrm{a} & 63 \pm 5 \mathrm{~b} & 95 \pm 11 \mathrm{a} & 93 \pm 17 \mathrm{a} & 76 \pm 13 \mathrm{ab} & 61 \pm 3 \mathrm{~b} & 67 \pm 7 \mathrm{~b} & 60 \pm 4 \mathrm{~b} \\ \mathrm{Cu}\left(\mathrm{mg} \mathrm{kg}^{-1}\right) & 11.5 \pm 1.8 \mathrm{a} & 10.0 \pm 0.6 \mathrm{a} & 10.9 \pm 0.7 \mathrm{a} & 9.9 \pm 2.5 \mathrm{ab} & 12.1 \pm 0.5 \mathrm{a} & 9.1 \pm 0.1 \mathrm{ab} & 8.9 \pm 0.3 \mathrm{~b} & 8.5 \pm 0.4 \mathrm{~b} \\ \mathrm{Na}\left(\mathrm{g} \mathrm{kg}^{-1}\right) & 5.0 \pm 0.4 \mathrm{bc} & 7.6 \pm 0.5 \mathrm{a} & 4.1 \pm 0.4 \mathrm{c} & 6.4 \pm 1.1 \mathrm{ab} & 0.8 \pm 0.0 \mathrm{~d} & 6.4 \pm 0.2 \mathrm{ab} & 5.5 \pm 1.3 \mathrm{bc} & 3.8 \pm 0.5 \mathrm{c} \\ \mathrm{Cl}\left(\mathrm{g} \mathrm{kg}^{-1}\right) & 8.3 \pm 0.4 \mathrm{a} & 8.9 \pm 0.5 \mathrm{a} & 5.6 \pm 0.5 \mathrm{~b} & 6.8 \pm 1.8 \mathrm{abc} & 2.0 \pm 0.5 \mathrm{~d} & 4.3 \pm 0.2 \mathrm{c} & 8.4 \pm 1.1 \mathrm{a} & 2.5 \pm 0.4 \mathrm{~d} \\ \mathrm{~Pb}\left(\mathrm{mg} \mathrm{kg}^{-1}\right) & 7.7 \pm 0.1 \mathrm{~d} & 8.9 \pm 0.3 \mathrm{ab} & 9.1 \pm 0.2 \mathrm{a} & 8.3 \pm 0.1 \mathrm{c} & 8.1 \pm 0.1 \mathrm{~cd} & 8.4 \pm 0.1 \mathrm{bc} & 8.1 \pm 0.2 \mathrm{~cd} & 7.7 \pm 0.6 \mathrm{~d}\end{array}$

pronounced variation between sites (Table 3) with the highest concentration in TF1 and the lowest in TF2 (Table 2). Variation in soil microelement concentrations (Fe, $\mathrm{Cu}, \mathrm{Zn}, \mathrm{Mn}, \mathrm{B})$ was relatively less pronounced than that for macroelements $\mathrm{K}, \mathrm{Ca}, \mathrm{Mg}$ and S (Table 3). Soil in site TF1 contained the highest concentration of $\mathrm{Zn}, \mathrm{Cu}$ and $\mathrm{B}$, and the second highest concentration of Fe (Table 2). The highest levels of Mn occurred in TF5 and TF7, and TF7 also had the highest concentration of Fe (Table 2). The highest concentration of the nonbiogenous heavy metal, $\mathrm{Pb}$, was found in TF1, followed by TF5 and TF7 (Table 2).Variation in concentration of $\mathrm{Na}$ and $\mathrm{Cl}$ was high (Table 3 ) and in general it reflected EC values in the sites, with the highest levels in TF1, followed by TF5 and TF6 (Table 2).

Mineral element concentrations were measured separately for leaf blades (Table 4) and leaf petioles of $T$. fragiferum plants (Table 5). For both macroelements and microelements, variation in tissue concentration was more pronounced in leaf petioles in comparison to that in leaf blades (Table 3). Concentration of both $\mathrm{Na}$ and $\mathrm{Cl}$ in plant tissues among different sites was relatively variable (Table 3 ), but it did not reflect variation in soil concentration of these elements (Table 4, Table 5). For example, the highest $\mathrm{Na}$ concentration in leaf petioles was found in plants growing in sites TF2, TF3, TF5 and TF6 (Table 5), where soil $\mathrm{Na}$ concentrations were 57, 64, 357 and $484 \mathrm{mg} \mathrm{L}^{-1}$, respectively (Table 2). However, in site TF1 with soil Na concentration reaching $1114 \mathrm{mg} \mathrm{L}^{-1}$, $\mathrm{Na}$ concentration in leaf petioles was significantly lower.

Plant-available concentration of mineral elements in soil as well as soil EC and $\mathrm{pH}$ of different $T$. fragiferum sites and concentration of elements in both leaf blades and leaf petioles were used for principal component analysis (Fig. 2) and to generate a heat map and hierarchial clusters showing similarity between different sites as well as various parameters (Fig. 3). Multivariate analysis generated an eight-component model explaining $100 \%$ of the total variance (Appendix 1). The first two components

Table 5. Mineral element concentrations in leaf petioles of Trifolium fragiferum from different sites. Data are means \pm SE from 3 samples from each site. Different letters within rows indicate statistically significant differences $(p<0.05)$

\begin{tabular}{|c|c|c|c|c|c|c|c|c|}
\hline $\begin{array}{l}\text { Element } \\
\text { (unit) }\end{array}$ & TF1 & TF2 & TF2b & TF3 & TF4 & TF5 & TF6 & TF7 \\
\hline $\mathrm{N}\left(\mathrm{g} \mathrm{kg}^{-1}\right)$ & $22.7 \pm 1.3 \mathrm{ab}$ & $20.8 \pm 0.3 b$ & $24.9 \pm 0.5 \mathrm{a}$ & $21.5 \pm 0.4 b$ & $15.8 \pm 0.3 \mathrm{~d}$ & $17.3 \pm 0.3 c$ & $21.0 \pm 0.5 b$ & $25.3 \pm 0.6 \mathrm{a}$ \\
\hline$P\left(\mathrm{~g} \mathrm{~kg}^{-1}\right)$ & $2.9 \pm 0.2 \mathrm{a}$ & $1.7 \pm 0.3 c$ & $1.3 \pm 0.1 \mathrm{~cd}$ & $1.8 \pm 0.4 \mathrm{bc}$ & $2.6 \pm 0.2 \mathrm{ab}$ & $1.1 \pm 0.1 \mathrm{~d}$ & $1.7 \pm 0.2 \mathrm{c}$ & $2.1 \pm 0.1 \mathrm{~b}$ \\
\hline $\mathrm{K}\left(\mathrm{g} \mathrm{kg}^{-1}\right)$ & $31 \pm 4 \mathrm{a}$ & $28 \pm 3 a$ & $30 \pm 2 \mathrm{a}$ & $19 \pm 8 b$ & $29 \pm 2 a$ & $15 \pm 1 c$ & $17 \pm 2 b c$ & $18 \pm 2 b$ \\
\hline $\mathrm{Ca}\left(\mathrm{g} \mathrm{kg}^{-1}\right)$ & $13.4 \pm 0.7 \mathrm{~b}$ & $1.8 \pm 0.4 \mathrm{e}$ & $12.3 \pm 0.5 b c$ & $9.8 \pm 0.7 \mathrm{~d}$ & $9.3 \pm 0.8 \mathrm{~d}$ & $15.4 \pm 0.1 \mathrm{a}$ & $11.3 \pm 0.9 \mathrm{bc}$ & $10.6 \pm 0.8 \mathrm{~cd}$ \\
\hline $\operatorname{Mg}\left(\mathrm{g} \mathrm{kg}^{-1}\right)$ & & & & & $4.7 \pm 0.4 \mathrm{ab}$ & & & $5.6 \pm 0.4 \mathrm{a}$ \\
\hline $\mathrm{Fe}\left(\mathrm{mg} \mathrm{kg}^{-1}\right)$ & $51 \pm 4 b$ & $48 \pm 5 \mathrm{bc}$ & $38 \pm 4 \mathrm{~cd}$ & & & & $115 \pm 5 a$ & $41 \pm 33 c$ \\
\hline $\operatorname{Mn}\left(\mathrm{mg} \mathrm{kg}^{-1}\right)$ & $25 \pm 2 c$ & $20 \pm 2 c$ & $22 \pm 1 c$ & $19 \pm 5 c$ & $24 \pm 2 c$ & $40 \pm 4 b$ & $67 \pm 5 a$ & $20 \pm 2 c$ \\
\hline $\mathrm{Zn}\left(\mathrm{mg} \mathrm{kg}^{-1}\right)$ & $53 \pm 5 \mathrm{a}$ & $37 \pm 4 \mathrm{bc}$ & $56 \pm 4 \mathrm{a}$ & $52 \pm 9 \mathrm{ab}$ & $39 \pm 3 b$ & $27 \pm 1 c$ & $27 \pm 2 c$ & $31 \pm 3 c$ \\
\hline $\mathrm{Cu}\left(\mathrm{mg} \mathrm{kg}^{-1}\right)$ & $9.0 \pm 0.6 \mathrm{a}$ & $7.5 \pm 0.7 b$ & $8.2 \pm 0.9 \mathrm{ab}$ & $7.7 \pm 2.0 \mathrm{ab}$ & $9.0 \pm 0.8 \mathrm{a}$ & $6.8 \pm 1.0 \mathrm{~b}$ & $6.2 \pm 0.4 b$ & $6.5 \pm 0.5 b$ \\
\hline $\mathrm{Na}\left(\mathrm{g} \mathrm{kg}^{-1}\right)$ & $13.7 \pm 0.5 b$ & $20.9 \pm 0.9 \mathrm{a}$ & $13.2 \pm 0.8 b$ & $19.5 \pm 1.6$ & $1.3 \pm 0.1 \mathrm{~d}$ & $23.5 \pm 1.0 \mathrm{a}$ & $21.9 \pm 1.4 \mathrm{a}$ & $8.8 \pm 0.7 \mathrm{c}$ \\
\hline $\mathrm{Cl}\left(\mathrm{g} \mathrm{kg}^{-1}\right)$ & $40.0 \pm 1.7 \mathrm{~b}$ & $53.0 \pm 3.0 \mathrm{a}$ & $40.5 \pm 1.9 \mathrm{~b}$ & $45.0 \pm 2.7 \mathrm{ab}$ & $12.8 \pm 0.9 \mathrm{~d}$ & $37.8 \pm 2.8 \mathrm{~b}$ & $39.0 \pm 2.6 b$ & $18.3 \pm 1.1 \mathrm{c}$ \\
\hline $\mathrm{Pb}\left(\mathrm{mg} \mathrm{kg}^{-1}\right)$ & $9.9 \pm 0.2 \mathrm{ab}$ & $10.6 \pm 0.3 \mathrm{a}$ & $9.8 \pm 0.3 \mathrm{abc}$ & $10.0 \pm 0.1 \mathrm{a}$ & $9.1 \pm 0.4 b$ & $10.4 \pm 0.1 \mathrm{a}$ & $10.8 \pm 0.7 \mathrm{a}$ & $8.6 \pm 0.4 c$ \\
\hline
\end{tabular}




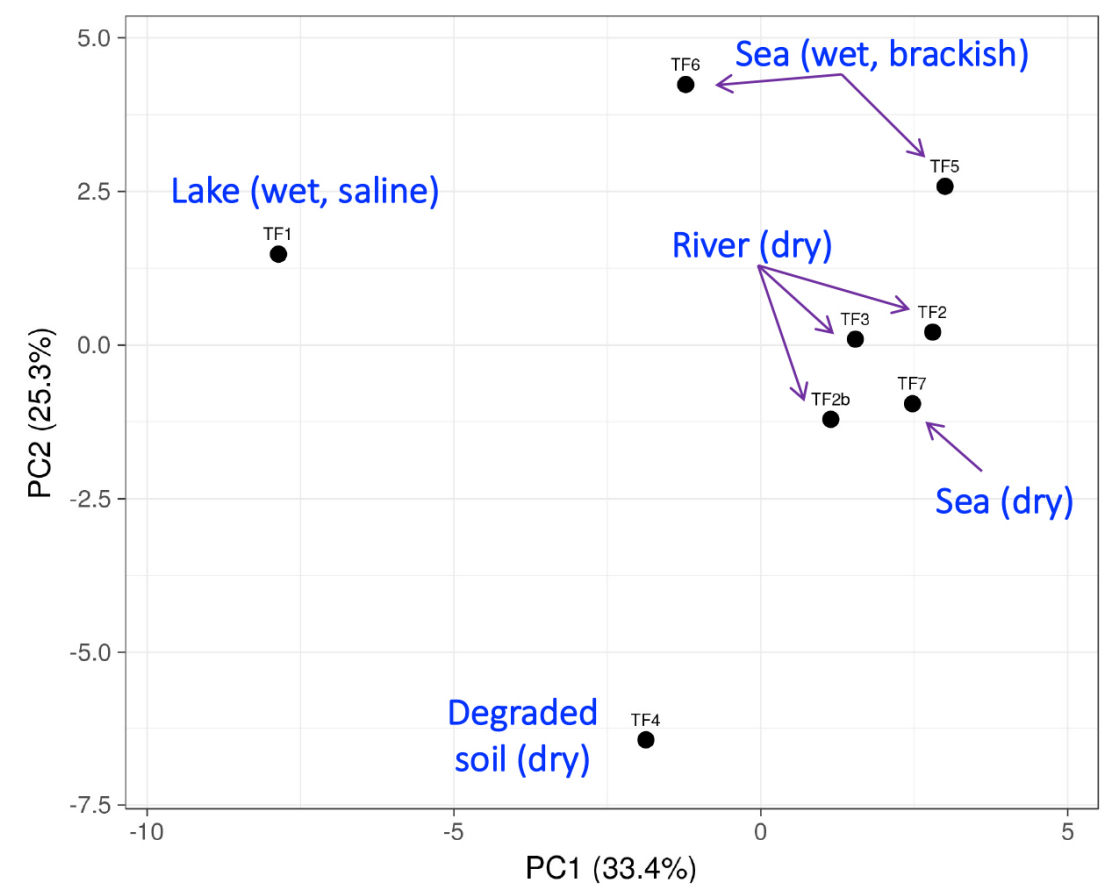

Fig. 2. Principal component analysis of soil characteristics, soil plant-available mineral element concentrations and element concentrations in leaf blades and leaf petioles in sites of Trifolium fragiferum. Unit variance scaling was applied to rows; singular value decomposition with imputation was used to calculate principal components. X and $\mathrm{Y}$ axis show principal component 1 and principal component 2 that explain $33.4 \%$ and $25.3 \%$ of the total variance, respectively.

represented $58.7 \%$ of the variance, and the obtained scatter plot showed clustering of TF2, TF2b, TF3 and TF7, with some association of TF5; and clear separation of TF1, TF4, and TF6 (Fig. 2). The highest loadings on the first component (PC1) were for soil $\mathrm{Zn}, \mathrm{Pb}$ and $\mathrm{Cu}$ concentration $(-0.26)$ followed by soil $\mathrm{S}$ concentration and Fe concentration in leaf blades (-0.25). High loadings were also for soil $\mathrm{EC}, \mathrm{Cl}, \mathrm{Na}$, and $\mathrm{B}$ concentration, and leaf blade $\mathrm{K}$ and petiole $\mathrm{P}$ concentration $(-0.24$ to -0.21$)$. The highest positive loading was for leaf blade $\mathrm{Mg}$ concentration (0.21). On the second component (PC2), the highest loading was for $\mathrm{Na}$ concentration in leaf blades and petioles $(-0.27$ and -0.24 , respectively), soil $\mathrm{Mg}$, $\mathrm{Ca}$ and $\mathrm{K}$ concentration ( 0.26 , 0.25 and 0.22 , respectively), followed by $\mathrm{Pb}$ concentration in leaf petioles $(-0.23)$, soil $\mathrm{K}$ concentration $(0.22), \mathrm{Cl}$ concentration in leaf blades and petioles, as well as $\mathrm{Mn}$ concentration in leaf petioles $(-0.20)$, and $\mathrm{Mg}$ and $\mathrm{Cu}$ concentration in leaf petioles $(0.20)$.

According to the heatmap and cluster analysis, there was a large difference between individual sites, as each of them had a rather unique soil mineral element and leaf tissue mineral element profile. The tightest association was evident between the three T. fargiferum sites related to River Lielupe (TF2, TF2b, TF3), but the site TF2, located closest to the seashore, somewhat differed from the other two. Two geographically adjacent sites located in wet coastal meadow (TF5, TF6) showed the next less close relationship, with TF7 displaying only negligible similarity with them. The remaining sites, TF1 and TF4, had rather unique profiles with only some degree of similarity between them as well as other sites.

Mineral element characteristics of soil and plant samples in different $T$. fragiferum sites showed relatively pronounced relationships (Fig. 3). First, a separate cluster was formed by soil $\mathrm{pH}$ and leaf blade concentration of $\mathrm{Mg}$ and $\mathrm{Pb}$. All other variables formed two major clusters with several subclusters each. Within the first cluster, four subclusters of variables were evident: (1) Ca concentration in leaf blades and petioles, and P concentration in soil; (2) Fe concentration in soil and leaf petioles, Mn concentration in leaf blades and petioles, and soil $\mathrm{N}$ concentration; (3) $\mathrm{N}$ concentration in leaf blades and petioles; and (4) $\mathrm{Na}$ and $\mathrm{Cl}$ concentration in leaf blades and petioles, as well as $\mathrm{Pb}$ concentration in leaf blades. Within the second cluster, the first association was formed by petiole $\mathrm{Mg}$ concentration, followed by soil Mn concentration, and very close association between soil $\mathrm{K}$ and $\mathrm{Ca}$ concentration. The remaining parameters formed two subgroups, where the first subgroup was formed by $\mathrm{Zn}$ concentration in leaf blades and petioles, leaf petiole $\mathrm{K}$ concentration and $\mathrm{Cu}$ concentration in leaf blades and petioles, which was followed by leaf blade $\mathrm{P}$ concentration, leaf blade $\mathrm{K}$ concentration, and leaf petiole $\mathrm{P}$ concentration. Within the second subgroup, leaf blade Fe concentration showed close association with soil $\mathrm{Cu}$ concentration, but the remaining characteristics exhibited extremely tight association, formed by $\mathrm{EC}$ and soil concentration of $\mathrm{Cl}, \mathrm{Na}, \mathrm{B}, \mathrm{Pb}, \mathrm{S}$, and $\mathrm{Zn}$. 


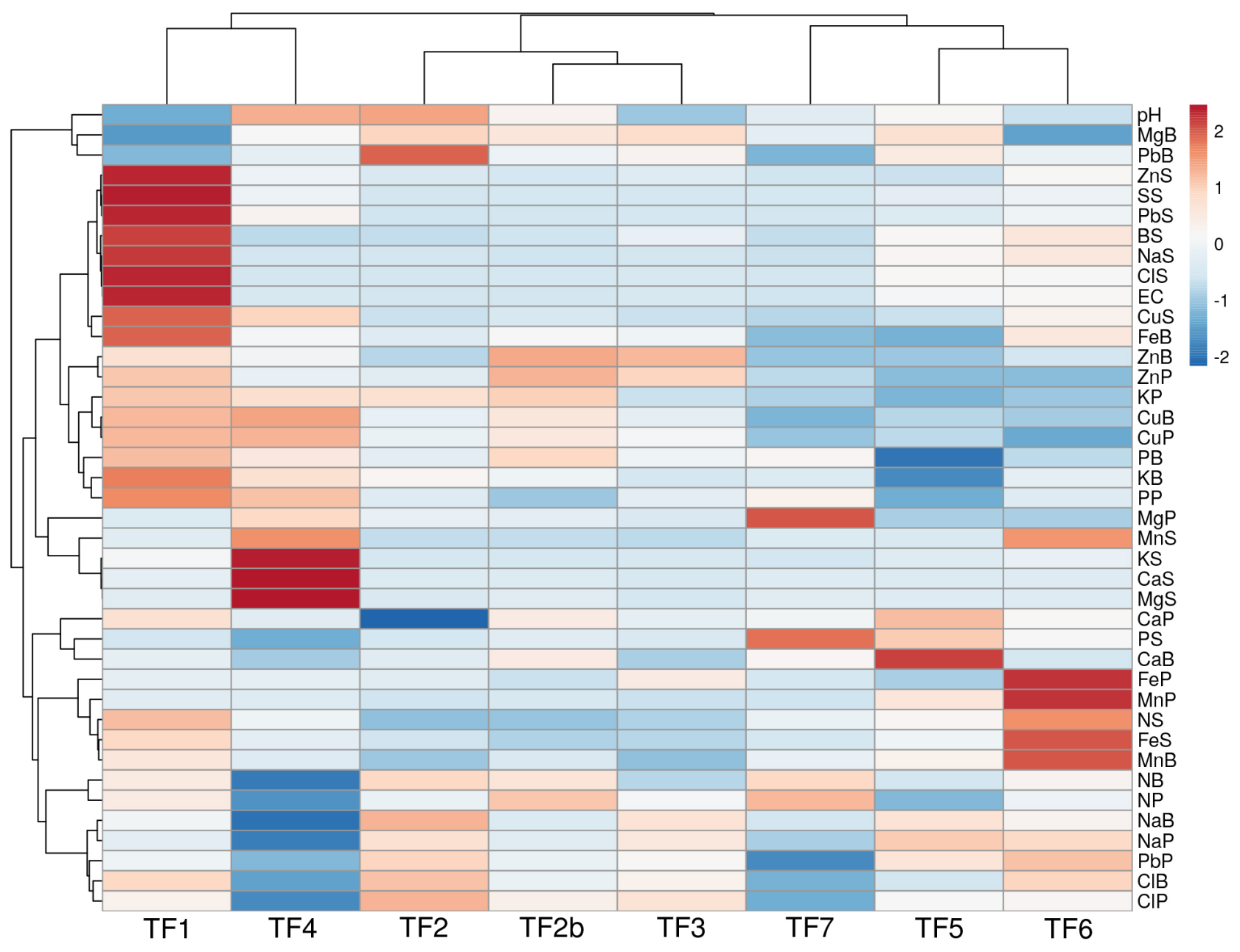

Fig. 3. Generated heat map and cluster analysis of variation in soil characteristics, soil plant-available mineral element concentrations (S) and element concentrations in leaf blades (B) and leaf petioles (P) in sites of Trifolium fragiferum. EC, electrical conductivity. Hierarchial clusters were generated by average linkage method with correlation distance. Color scale shows relative intensity of normalized parameter values.

\section{Discussion}

From a point of view of vegetation science, $T$. fragiferum is an important species for classification of saline and brackish grasslands of the Baltic Sea coast (Pätsch et al. 2019). In these habitats, the main variable environmental factors basically determining vegetation differences were soil salinity and moisture, and, to a lesser extent, nutrient availablity and soil $\mathrm{pH}$. As already observed in earlier studies in Sweden (Tyler 1969), T. fragiferum is characteristically located in oligohaline to mesohaline soils of the middle and upper geolittoral or on the transition zone from supralittoral to epilittoral also in other parts of the Baltic Sea coast (Pätsch et al. 2019). In these habitats, existence of specific adaptive mechanisms related to mineral nutrition of coastal plant species could be proposed, due to pronounced effect of seawater as well as organic debris-associated processes.

In Latvia, micropopulations of T. fragiferum appear also outside typical coastal grassland habitats or inland-located salt-affected grassland habitats, as in the case of TF2b and
TF4. Also, while TF7 was located in close vicinity to the sea, the habitat itself represented relatively intensively trampled roadside vegetation within a dry coastal meadow with a low salinity level (Table 2). The accession of $T$. fragiferum found in an urban territory (TF4) seemed to represent a special case, as this habitat was under high anthropogenic pressure, evidently being affected by use of artificial substrate for levelling the ground, and this was also reflected by substrate chemical composition, with extremely high concentration of plant-available $\mathrm{K}, \mathrm{Ca}$, and $\mathrm{Mg}$, high $\mathrm{pH}$ and relatively low EC (Table 2). This micropopulation most likely represents remnants from the pre-urbanic period, when the particular site was located within the estuary of River Daugava.

It can be proposed that association of $T$. fragiferum with shores of different waterbodies (lake and sea) and rivers is related not to requirement for increased soil salinity but rather to deposition of waterborne organic debris on grassland. In addition, this also points to possible spread of propagules by water. T. fragiferum stands out among other clover species due to development of fruiting heads 
(Zohary, Heller 1982). After flowering, inflorescences (flower heads) develop into fruiting heads consisting of inflated fruit capsules formed from calyx structures, being significantly larger than the flower heads. Fruiting heads have significant floating ability and their formation can be considered as adaptation for seed dispersal. Indeed, it is accepted that $T$. fragiferum is dispersed by water (Tyler et al. 2021). Most likely, deposit of nitrogen-rich organic material (phytodetritus) acts as a nutrient source during plant establishment, and it can also carry plant propagules (Wolters et al. 2017).

Edaphic conditions in coastal habitats are known to be highly variable, with soil mineral nutrient concentrations showing both temporal and spatial heterogeneity (Karlsons et al. 2017). Therefore, one of the most important adaptive fetaures of wild coastal plant species is related to ability to tolerate wide range of plant-available concentrations of essential mineral nutrients without much changes in physiological status and biomass accumulation rate (Ievinsh 2006). Variation in plant-available mineral nutrient concentration in soil of different sites with T. fragiferum was relatively higher as that found for sites with another coastalspecific rare plant species, Triglochin maritima (Karlsons et al 2011). Only relatively slightly higher variation among $T$. fragiferum soils was found for N, P and S; but more higher variation was evident for $\mathrm{K}, \mathrm{Ca}, \mathrm{Mg}$ and all micronutrients. Most probably, these differences reflect a wider range of adaptation of $T$. fragiferum to various habitats with more diverse soil conditions in comparison to T. maritima, which is a species preferring saline wetlands and is considered as an indicator species of coastal meadows in the Baltic Sea region (Rūsiņa 2013). In contrast, T. fragiferum was found on both saline and non-saline soils, including also dry coastal meadow as well as degraded urban land as native habitats of the species (Table 1).

Due to efficient $\mathrm{N}_{2}$-fixing ability of rhziobial symbiosis, T. fragiferum plants seem to be able to succeed also on soils with relatively low plant-available $\mathrm{N}$ concentration, as in the case of sites TF2, TF2b and TF3 (Table 2). Leaf blade $\mathrm{N}$ concentrations of these plants reached optimum or close to optimum level (Table 3). While T. fragiferum soils were relatively rich in plant-available $\mathrm{P}$ (Table 2), the concentration range of this element in leaf blades was lower than the proposed optimum for T. repens (Table 3). Leaf blade $\mathrm{K}$ concentrations for plants in all sites were outside the estimated optimum range, and soil concentrations were lower than those proposed as optimal for legumes. All other macronutrients were abundant in T. fragiferum soils, and concentration of these elements in leaf tissues was at optimum or above optimum levels. Thus, Ca in T. fragiferum soils showed luxury supply, and this was reflected in optimal or even above optimum concentration levels of $\mathrm{Ca}$ in leaves. Another macronutrient, $\mathrm{Mg}$, had similarly high soil concentrations in all sites and higher than optimum concentration in leaves. Similarly, soil micronutrient concentrations in $T$. fragiferum sites were above optimum level (Table 3). However, the leaf concentration range was variable for different micronutrients: it was below optimum for $\mathrm{Cu}$, optimum for $\mathrm{Fe}$, and above optimum for $\mathrm{Mn}$. Accumulation of surplus levels of metals in leaf tissues of T. fragiferum, which was especially pronounced for $\mathrm{Mg}$, $\mathrm{Zn}$, and $\mathrm{Mn}$, could be related to avoidance from excess metal, most probably, by sequestration of chelated metals in cell vacuoles (Peng, Gong 2014). It needs to be pointed out that optimum levels of mineral concentrations in wildgrown plant tissues could differ from these established for cultivated plants, and mineral nutrient needs of $T$. fragiferum could differ from these of $T$. repens, which were used for comparison (Schwab et al. 2007).

Soil $\mathrm{pH}$ ranged from slightly acidic $(\mathrm{pH}$ 6.53) to alkaline ( $\mathrm{pH} 8.70)$. With soil $\mathrm{pH}$ reaching 8.7 , as in the case of T. fragiferum sites TF2 and TF4 (Table 2), availability of several elements for plants significantly decreases (Rengel 2002). However, the only indication of possible mineral deficiency in T. fragiferum plants growing on alkaline soils was an extremely low $\mathrm{Ca}$ concentration in leaf petioles of plants at TF2 (Table 5).

Multivariate analysis showed that sites with similar localization (TF2, TF2b, TF3 on river shores or TF5, TF6, TF7 in coastal areas) indeed had similar characteristics, as these grouped together according to both soil mineral nutrient availability as well as leaf tissue mineral element concentration (Fig. 3). In contrast, site TF1, located on a salt-affected wet meadow on a lake shore and site TF4 on degraded urban land, displayed strikingly different sets of soil and plant nutrient characteristics. It is evident that, due to generally higher variability in plant-available mineral elements in soils in comparison to those in plant tissues, the relative impact of soil characteristics on results of multivariate analysis was more pronounced. Relatively strong association found between several soil element concentrations across T. fragiferum sites (Fig. 3) points to interaction between soil mineral nutrient dynamics and other prevalent environmental factors. Especially pronounced association was evident between soil EC, $\mathrm{Na}$, $\mathrm{Cl}, \mathrm{B}, \mathrm{S}$ and $\mathrm{Zn}$ concentrations, which probably reflects the influence of seawater inundation on soils in T. fragiferum sites. Concomitant changes in concentration of plantavailable mineral nutrients was already shown to occur in a saline water-affeted wet grassland, where $T$. fragiferum was also present together with characteristic halophytic species (Karlsons et al. 2017).

In conclusion, $T$. fragiferum can be characterized as a species able to grow in variety of water shore-associated habitats and able to maintain mineral homeostasis in leaf tissues irrespective of large variability in soil plantavailable mineral nutrient concentrations and different levels of soil pH. From a conservation perspective of CWR species, flexibility in mineral nutrition of $T$. fragiferum allows to recognize the species as tolerant to anthropogenic pressure in form of environmental contamination with mineral compunds. Together with general resilience to 
environmental constraints, this allows to predict a relatively good conservation status of the species in Latvia in spite of rather limited number and size of the geographically isolated micropopulations of $T$. fragiferum.

\section{Acknowledgements}

The study was supported by the Latvian Science Council project lzp-2020/2-0349 "Molecular, physiological and ecological evaluation of Latvian genetic resources of valuable wild legume species, Trifolium fragiferum, in a context of sustainable agriculture". Participation of Magnolia Garbarino (Pawling High School, NY, USA) in the field work is sincerely acknowledged.

\section{References}

Andersone-Ozola U., Jēkabsone A., Purmale L., Romanovs M., Ievinsh G. 2021. Abiotic stress tolerance of coastal accessions of a promising forage species, Trifolium fragiferum. Plants 10: 1552.

Cabinet of Ministers of Latvia. 2000. Provisions on the List of Specially Protected Species and Species of restricted Use. Regulations of the Cabinet of Ministers of Latvia No. 396, 2000.

Can E., Arslan M., Sener O., Daghan H. 2013. Response of strawberry clover (Trifolium fragiferum L.) to salinity stress. Res. Crops 14: 576-584.

Dūmiņš K., Andersone-Ozola U., Samsone I., Elferts D., Ievinsh G. 2021. Growth and physiological performance of a coastal species Trifolium fragiferum as affected by a coexistence with Trifolium repens, $\mathrm{NaCl}$ treatment and inoculation with rhyzobia. Plants 10: 2196.

Egan L.M., Hofmann R.W., Ghamkhar K., Hoyos-Villegas V. 2021. Prospects for Trifolium improvement through germplasm characterization and pre-breeding in New Zealand and beyond. Front. Plant Sci. 12: 653191.

FAO. 2009. International Treaty on Plant Genetic Resources for Food and Agriculture. Food and Agriculture Organization of the United Nations, Rome.

Ford-Lloyd B.V., Schmidt M., Armstrong S.J., Barazani O., Engels J., Hadas R., Hammer K., Kell S.P., Kang D., Khoshbakht K., Li Y., Long C., Lu B.-R., Ma K., Nguyen V.T., Qiu L., Ge S., Wei W., Zhang Z., Maxted N. 2011. Crop wild relatives: Undervalued, underutilized and under threat? Bioscience 61: 559-565.

Haswell S.J. 1991. Atomic Absorption Spectrometry: Theory, Design and Applications. Elsevier, Amsterdam.

Huber H., Wiggerman L. 1997. Shade avoidance in the clonal herb Trifolium fragiferum: A field study with experimentally manipulated vegetation height. Plant Ecol. 130: 53-62.

Ievinsh G. 2006. Biological basis of biological diversity: physiological adaptations of plants to heterogeneous habitats along a sea coast. Acta Univ. Latv. 710: 53-79.

Ievinsh G., Ievina S., Andersone-Ozola U., Samsone I. 2021. Leaf sodium, potassium and electrolyte accumulation capacity of plant species from salt-affected coastal habitats of the Baltic Sea: Towards a definition of $\mathrm{Na}$ hyperaccumulation. Flora 274: 151748.

Karlsons A., Druva-Lusite I., Necajeva J., Andersone-Ozola U., Samsone I., Osvalde A., Ievinsh G. 2017. Adaptation strategies of rare plant species to heterogeneous soil conditions on a coast of a lagoon lake as revealed by analysis of mycorrhizal symbiosis and mineral constituent dynamics. Environ. Exp. Biol. 15: 113-126.

Karlsons A., Osvalde A., Ievinsh G. 2011. Growth and mineral nutrition of two Triglochin species from saline wetlands: adaptation strategies to conditions of heterogeneous mineral supply. Environ. Exp. Biol. 9: 83-90.

Maxted N. 2003. Conserving the genetic resources of crop wild relatives in European protected areas. Biol. Conserv. 113: 411-417.

Maxted N., Ford-Lloyd B.V., Jury S., Kell S., Scholten M. 2006. Towards a definition of a crop wild rerlative. Biodiv. Conserv. 15: 2673-2685.

Maxted N., Scholten M., Codd R., Ford-Lloyd B. 2007. Creation and use of a national inventory of crop wild relatives. Biol. Conserv. 140: 142-159.

Metsalu T., Vilo J. 2015. ClustVis: a web tool for visualizing clustering of multivariate data using Principal Component Analysis and heatmap. Nucl. Acids Res. 43: W566-W570.

Nichols P.G.H., Revell C.K., Humphries A.W., Howie J.H., Hall E.J., Sandral G.A., Ghamkhar K., Harris C.A. 2012. Temperate pasture legumes in Australia - their history, current use, and future prospects. Crop Pasture Sci. 63: 691-725.

Osvalde A. Optimization of plant mineral nutrition revisited: the roles of plant requirements, nutrient interactions, and soil properties in fertilization management. Environ. Exp. Biol. 9: $1-8$.

Patnaik P. 1997. Handbook of Environmental Analysis. CRC Press, New York. 584 p.

Pätsch R., Schaminée J.H.J., Janssen J.A.M., Hennekens S.M., Bruchmann I., Jutila H., Meisert A., Bergmeier E. 2019. Between land and sea - a classification of saline and brackish grasslands of the Baltic Sea coast. Phytocenologia 49: 319-348.

Peng J.-S., Gong J.-M. 2014. Vacuolar sequestration capacity and long-distance metal transport in plants. Front. Plant Sci. 5: 19.

Prohens J., Gramazio P., Plazas M., Dempewolf H., Kilian B., Díez M.J., Fita A., Herraiz F.J., Rodríguez-Burruezo A., Soler S., Knapp S., Vilanova S. 2017. Introgressiomics: a new approach for using crop wild relatives in breeding for adaptation to climate change. Euphytica 213: 158.

Ravaagnani A., Abberton M.T., Skøt L. 2012. Development of genomic resources in the species of Trifolium L. and its application in forage legume breeding. Agronomy 2: 116-131.

Rengel Z. 2002. Habdbook of Plant Growth: $p H$ as the Master Variable. CRC Press, New York. 472 p.

Rinkis G., Ramane H., Kunicka T. 1987. Methods of Soil and Plant Analysis. Zinatne, Riga. /in Russian/

Rūsina S. 2013. 1630* Boreal Baltic coastal meadows. In: Auniņš A. (Ed.) European Union Protected Habitats in Latvia. Interpretation Manual. Riga, Latvian Fund for Nature, Ministry of Environmental Protection and Regional Development, pp. 55-57.

Schwab G.J., Lee C.D., Pearce R., Thom W.O. 2007. Sampling Plant Tissue for Nutrient Analysis. Cooperative Extension Service, University of Kentucky.

Sreenivasulu V., Kumar N.S., Dharmendra V., Asif M., Balaram V., Zhengxu H., Zhen Z. 2017. Determination of boron, phosphorus, and molybdenum content in biosludge samples by microwave plasma atomic emission spectrometry (MPAES). Appl. Sci. 7: 264.

Tyler G. 1969. Regional aspects of Baltic shore-meadow vegetation. Vegetatio 19: 60-86. 
Tyler T., Herbertsson L., Olofsson J., Olsson P.A. 2021. Ecological indicator and traits values for Swedish vascular plants. Ecol. Indic. 120: 106923.

Warschefsky E., Penmetsa R.V., Cook D.R., vonWettberg E.J.B. 2014. Back to the wilds: Tapping evolutionary adaptations for resilient crops through systematic hybridization with crop wild relatives. Am. J. Bot. 101: 1791-1800.

White P.J., Brown P.H. 2010. Plant nutrition for sustainable development and global health. Ann. Bot.105: 1073-1080.

Wolters M., de Vries S., Ozinga W., Bakker J.P. 2017. Restoration of inland brakish vegetation by large-scale transfer of coastal driftline material. Appl. Veget. Sci. 20: 641-650.

Zhang H., Mittal N., Leamy L.J., Barazani O., Song B.-H. 2016. Back into the wild - Apply untapped genetic diversity of wild relatives for crop improvement. Evol. Applic. 10: 5-24.

Zhang H., Yasmin F., Song, B.-H. 2019. Neglected treasures in the wild - legume wild relatives in food security and human health. Curr. Opin. Plant Biol. 49: 17-26.

Zohary M., Heller D. 1984. The Genus Trifolium. Israel Academy of Sciences and Humanities. 606 p.

Appendix 1. Values for loadings of principal components during mulivariate analysis of chemical element concetration in soils and plant tissues of Trifolium fragiferum at various sites. S, soil concentration; B, concentration in leaf blade; P, concentration in leaf petiole; EC, electrical conductivity

\begin{tabular}{|c|c|c|c|c|c|c|c|c|}
\hline Parameter & PC1 & PC2 & PC3 & PC4 & PC5 & PC6 & PC7 & PC8 \\
\hline NS & -0.1772 & -0.1468 & -0.2344 & 0.0155 & 0.0216 & 0.0240 & 0.0128 & -0.8341 \\
\hline PS & 0.1287 & -0.1312 & -0.1277 & 0.3279 & -0.0292 & 0.1053 & -0.0146 & 0.1002 \\
\hline KS & -0.1071 & 0.2244 & -0.1771 & -0.1643 & -0.0686 & 0.0020 & -0.0302 & 0.0421 \\
\hline $\mathrm{CaS}$ & -0.0712 & 0.2543 & -0.1663 & -0.1416 & -0.0555 & 0.0233 & 0.0008 & 0.2217 \\
\hline $\mathrm{MgS}$ & -0.0639 & 0.2565 & -0.1725 & -0.1306 & -0.0603 & 0.0068 & 0.0333 & -0.1638 \\
\hline SS & -0.2537 & -0.0558 & 0.0399 & 0.0605 & -0.1256 & 0.1382 & -0.0613 & -0.1790 \\
\hline $\mathrm{FeS}$ & -0.1518 & -0.1828 & -0.2043 & -0.0900 & 0.1171 & 0.0047 & 0.0940 & 0.1579 \\
\hline $\mathrm{MnS}$ & -0.0936 & 0.0594 & -0.3072 & -0.1813 & 0.1525 & -0.0710 & 0.1096 & 0.0332 \\
\hline $\mathrm{ZnS}$ & -0.2622 & -0.0591 & 0.0550 & 0.0235 & -0.0219 & 0.0936 & -0.0874 & 0.0223 \\
\hline $\mathrm{CuS}$ & -0.2666 & 0.0355 & -0.0482 & -0.0548 & -0.0394 & 0.0546 & 0.0240 & 0.0476 \\
\hline BS & -0.2176 & -0.1746 & 0.0148 & 0.0304 & -0.1232 & 0.0121 & -0.1156 & 0.0676 \\
\hline $\mathrm{NaS}$ & -0.2267 & -0.1544 & -0.0055 & 0.0284 & -0.1338 & 0.1036 & -0.0172 & 0.0555 \\
\hline ClS & -0.2275 & -0.1319 & 0.0394 & 0.0656 & -0.1658 & 0.1165 & -0.0576 & 0.0507 \\
\hline $\mathrm{PbS}$ & -0.2632 & -0.0239 & 0.0101 & 0.0360 & -0.1066 & 0.1191 & -0.0752 & 0.0154 \\
\hline NB & 0.0219 & -0.1575 & 0.1565 & 0.1934 & 0.2476 & 0.2602 & 0.3556 & 0.0669 \\
\hline $\mathrm{PB}$ & -0.1545 & 0.1582 & 0.1814 & 0.1256 & 0.2068 & -0.0642 & 0.1397 & 0.0231 \\
\hline $\mathrm{KB}$ & -0.2254 & 0.0991 & 0.1074 & -0.0201 & 0.1853 & 0.1605 & 0.0541 & -0.0214 \\
\hline $\mathrm{CaB}$ & 0.0994 & -0.1157 & -0.0425 & 0.1411 & -0.4257 & 0.1176 & 0.3401 & -0.0322 \\
\hline $\mathrm{MgB}$ & 0.2124 & 0.1071 & 0.1460 & -0.0965 & -0.1872 & -0.0155 & -0.0719 & -0.1167 \\
\hline $\mathrm{FeB}$ & -0.2510 & -0.0292 & 0.1019 & -0.0845 & 0.1062 & -0.0973 & 0.0484 & 0.0556 \\
\hline $\mathrm{MnB}$ & -0.1193 & -0.1781 & -0.2448 & -0.0047 & 0.0719 & -0.0490 & 0.2862 & 0.0993 \\
\hline $\mathrm{ZnB}$ & -0.0992 & 0.0697 & 0.2336 & -0.0150 & -0.0265 & -0.4909 & 0.0491 & -0.2104 \\
\hline $\mathrm{CuB}$ & -0.1789 & 0.1838 & 0.1220 & -0.1244 & -0.1278 & -0.0277 & 0.1435 & 0.0285 \\
\hline $\mathrm{NaB}$ & 0.0862 & -0.2399 & 0.1716 & -0.1008 & -0.0258 & 0.1348 & -0.2181 & 0.0280 \\
\hline $\mathrm{ClB}$ & -0.0731 & -0.2065 & 0.1886 & -0.2007 & 0.1700 & 0.0655 & -0.0098 & 0.0114 \\
\hline $\mathrm{PbB}$ & 0.1486 & -0.0288 & 0.1146 & -0.3488 & -0.0286 & 0.1940 & 0.0052 & -0.0885 \\
\hline $\mathrm{NP}$ & 0.0015 & -0.0751 & 0.1859 & 0.3119 & 0.2906 & -0.0825 & 0.2122 & -0.1064 \\
\hline PP & -0.2149 & 0.1260 & -0.0281 & 0.0696 & 0.1222 & 0.1809 & -0.3128 & 0.0396 \\
\hline $\mathrm{KP}$ & -0.1358 & 0.1542 & 0.2161 & -0.0879 & 0.0300 & 0.1467 & 0.3694 & -0.0147 \\
\hline $\mathrm{CaP}$ & -0.0796 & -0.0837 & -0.1257 & 0.2075 & -0.3336 & -0.3388 & 0.1731 & 0.1038 \\
\hline $\mathrm{MgP}$ & 0.0370 & 0.1999 & -0.0775 & 0.2794 & 0.1953 & 0.2021 & -0.1137 & -0.0497 \\
\hline $\mathrm{FeP}$ & -0.0617 & -0.1362 & -0.1389 & -0.1856 & 0.3639 & -0.2381 & -0.1184 & 0.0753 \\
\hline $\mathrm{MnP}$ & -0.0297 & -0.1963 & -0.2510 & -0.1449 & 0.0706 & -0.1264 & 0.2284 & 0.0493 \\
\hline $\mathrm{ZnP}$ & -0.1084 & 0.0831 & 0.3050 & 0.0363 & -0.0215 & -0.2871 & 0.0308 & 0.0447 \\
\hline $\mathrm{CuP}$ & -0.1601 & 0.1964 & 0.1624 & -0.0736 & -0.1590 & -0.0236 & 0.0411 & 0.0379 \\
\hline $\mathrm{NaP}$ & 0.0831 & -0.2735 & 0.0752 & -0.1448 & -0.0726 & -0.0108 & -0.0898 & 0.0456 \\
\hline $\mathrm{ClP}$ & 0.0289 & -0.1978 & 0.2583 & -0.1884 & 0.0006 & 0.0143 & 0.0047 & -0.0796 \\
\hline $\mathrm{PbP}$ & 0.0128 & -0.2264 & 0.0703 & -0.3130 & -0.0408 & 0.0005 & 0.1039 & 0.0065 \\
\hline
\end{tabular}

